\title{
A SIMPLE APPROACH TO THE SUMMATION OF CERTAIN SLOWLY CONVERGENT SERIES
}

\author{
STANISLAW LEWANOWICZ
}

\begin{abstract}
Summation of series of the form $\sum_{k=1}^{\infty} k^{\nu-1} r(k)$ is considered, where $0 \leq \nu \leq 1$ and $r$ is a rational function. By an application of the Euler-Maclaurin summation formula, the problem is reduced to the evaluation of Gauss' hypergeometric function. Examples are given.
\end{abstract}

\section{INTRODUCTION}

In a recent paper [3], Gautschi has considered series of the type

$$
\sum_{k=1}^{\infty} k^{\nu-1} r(k)
$$

where $0<\nu \leq 1$ and $r$ is a rational function

$$
r(k)=\frac{p(k)}{q(k)},
$$

$p, q$ being real polynomials, $\operatorname{deg} p \leq \operatorname{deg} q$. It is assumed that the zeros of $q$ all have nonpositive real parts. By obtaining the fraction decomposition of $r$, the problem can be simplified to considering rational functions of the form

$$
r(k)=\frac{1}{(k+a)^{m}} \quad(\Re a \geq 0, m \geq 1) .
$$

The fractional power $k^{\nu-1}$ in (1.1) may be generalized to $(k+b)^{\nu-1}, \Re b \geq 0$; we thus consider series of the type

$$
\sum_{k=1}^{\infty} \frac{(k+b)^{\nu-1}}{(k+a)^{m}} \quad(m \geq 1,0<\nu<1) .
$$

In the cited paper, the sum of the series (1.2) is expressed as a weighted integral over $\mathbb{R}_{+}$of certain special functions related to the incomplete gamma function. Gaussian quadrature is applied to the integral, using $w_{\nu}(t)=t^{-\nu} \varepsilon(t)$, where $\varepsilon(t)=t\left(e^{t}-1\right)^{-1}$ is the Einstein function, as a weight function on $[0, \infty)$. Convergence of the quadrature formula can be shown. Nevertheless,

Received by the editor December 30, 1992 and, in revised form, September 14, 1993.

1991 Mathematics Subject Classification. Primary 40A25; Secondary 65D30.

Key words and phrases. Slowly convergent series, Euler-Maclaurin formula, Gauss' hypergeometric function.

Supported by Komitet Badań Naukowych (Poland) under grant No. 210299101. 
the application of the method is not a simple task, in general. The main difficulty is connected with the evaluation of the incomplete gamma function. For $\nu=\frac{1}{2}$, this function is expressible in terms of Dawson's integral $F$, which can be computed to high accuracy.

In this paper, we show that the problem of summing the series (1.2) may be solved using very simple tools. Application of the Euler-Maclaurin formula reduces the problem to the evaluation of Gauss' hypergeometric function ${ }_{2} F_{1}$.

\section{RESULTS}

Let $s$ denote the sum of the series (1.2). We can write

$$
s=\sum_{k=1}^{n-1} \frac{(k+b)^{\nu-1}}{(k+a)^{m}}+r_{n},
$$

where

$$
r_{n}:=\sum_{k=n}^{\infty} \frac{(k+b)^{\nu-1}}{(k+a)^{m}}
$$

Application of the Euler-Maclaurin formula (see, e.g., [1, 23.1.30]) to (2.2) gives

$$
r_{n}-\int_{n}^{\infty} f(x) d x-\frac{1}{2} f(n) \sim-\sum_{k=1}^{\infty} \frac{B_{2 k}}{(2 k) !} f^{(2 k-1)}(n),
$$

where

$$
f(x):=\frac{(x+b)^{\nu-1}}{(x+a)^{m}}
$$

and $B_{j}$ is the $j$ th Bernoulli number. Using a formula of [4, 3.194.2], we obtain

$$
\int_{n}^{\infty} f(x) d x=\frac{1}{m-\nu}(n+b)^{\nu-m} F_{1}\left(\begin{array}{c|c}
m, m-\nu & \frac{b-a}{m+1-\nu} \\
m+b
\end{array}\right) .
$$

Equations (2.3), (2.4) and the formula

$$
f^{(r)}(x)=\frac{(-1)^{r}(1-\nu)_{r}}{(x+a)^{m}(x+b)^{r+1-\nu}} \sum_{j=0}^{r}(-1)^{j}\left(\begin{array}{l}
r \\
j
\end{array}\right) \frac{(m)_{j}}{(\nu-r)_{j}}\left(\frac{x+b}{x+a}\right)^{j} \quad(r \geq 0)
$$

imply that

$$
r_{n}=r_{n}^{(q)}+O\left(n^{-2 q-1}\right) \quad(q \geq 1),
$$

where

$$
\begin{aligned}
r_{n}^{(q)}= & \frac{(n+b)^{\nu-m}}{m-\nu}{ }_{2} F_{1}\left(\begin{array}{c}
m, m-\nu \\
m+1-\nu
\end{array} \mid \frac{b-a}{n+b}\right) \\
& +\frac{(n+b)^{1-\nu}}{(n+a)^{m}}\left\{\frac{1}{2}+\sum_{k=1}^{q} \frac{B_{2 k}(1-\nu)_{2 k-1}}{(2 k) !(n+b)^{2 k-1}} \sigma_{k}\right\} .
\end{aligned}
$$

Here,

$$
\sigma_{k}:=\sum_{j=0}^{2 k-1}(-1)^{j}\left(\begin{array}{c}
2 k-1 \\
j
\end{array}\right) \frac{(m)_{j}}{(\nu-2 k+1)_{j}}\left(\frac{n+b}{n+a}\right)^{j} \quad(k=1,2, \ldots),
$$


and $(c)_{j}:=c(c+1) \cdots(c+j-1)$ is Pochhammer's symbol.

The function ${ }_{2} F_{1}$ appearing in the equation (2.6) can be evaluated by applying some standard linear transformations (see [1, 15.3.4, 15.3.7]) which brings this function into a form having a fast convergent power series expansion.

\section{EXAMPLES}

We made several tests, taking various combinations of the parameters. In all examples below we take $q=4$ (cf. (2.5)), i.e., we compute the approximation

$$
\tilde{s}_{n}:=\sum_{k=1}^{n-1} \frac{(k+b)^{\nu-1}}{(k+a)^{m}}+r_{n}^{(4)}
$$

to the sum $s$ of the series (1.2), where $r_{n}^{(4)}$ is defined by (2.6).

All the computations were done on a personal 486-based computer, in extended arithmetic (about 18 decimal places).

Example 3.1. Let $a>0, b=0, \nu=\frac{1}{2}$. This is the case discussed in [3, §3]. Note that the hypergeometric function ${ }_{2} F_{1}(z)$ in the formula (2.6) simplifies in this case to the arctan function. The results for $m=1$ are shown in Table 3.1. Convergence is very fast, even for large $a$, in which case Gautschi's method of [3] needs some extra effort to achieve good accuracy. This remains true for other values of $\nu \in(0,1)$ and $b>0$; in Table 3.2 we give a selection of results obtained for $\nu=\frac{9}{10}, b=\frac{1}{2}$ and $m=1$.

A similar rate of convergence is observed for $m>1$.

TABLE 3.1. Approximations (3.1) to the sum of (1.2) for $a=1$,

$$
\begin{array}{rlll}
8,64, b=0, \nu=\frac{1}{2}, m=1 & \\
\multicolumn{1}{c}{n} & \multicolumn{1}{l}{a=1} & \multicolumn{1}{c}{a=8} & \multicolumn{1}{c}{a=64} \\
5 & 1.8600250788 & 0.93137293396 & 0.3699316982450 \\
10 & 1.8600250792207 & 0.93137293400304 & 0.369931698249664 \\
15 & 1.860025079221182 & 0.9313729340031025 & 0.3699316982496709 \\
20 & 1.8600250792211898 & 0.93137293400310378 & 0.36993169824967112 \\
30 & 1.86002507922119030 & 0.93137293400310387 & 0.36993169824967113 \\
40 & 1.86002507922119031 & 0.93137293400310387 &
\end{array}
$$

TABLE 3.2. Approximations (3.1) to the sum of (1.2) for $a=1$, $8,64, b=\frac{1}{2}, \nu=\frac{9}{10}, m=1$

\begin{tabular}{rlll}
$n$ & \multicolumn{1}{c}{$a=1$} & \multicolumn{1}{c}{$a=8$} & \multicolumn{1}{c}{$a=64$} \\
5 & 9.61542462126 & 8.1705856754462 & 6.6951505182819 \\
10 & 9.61542462140421 & 8.170585675449024 & 6.6951505182822286 \\
15 & 9.6154246214045207 & 8.17058567544903510 & 6.69515051828222948 \\
20 & 9.61542462140452727 & 8.17058567544903549 & 6.69515051828222951 \\
30 & 9.61542462140452771 & 8.17058567544903553 & 6.69515051828222951 \\
40 & 9.61542462140452772 & 8.17058567544903553 &
\end{tabular}

Example 3.2. The method works well also for complex $a$. For $a=i \alpha, \alpha>0$, $b=0, \nu=\frac{1}{2}, m=1$, we obtained the results shown in Table 3.3. For each $n$, the first entry is $\Re \tilde{s}_{n}$, the second $\Im \tilde{s}_{n}$ (cf. (3.1)). The nature of the convergence is the same as in the preceding examples. In contrast with the method of [3], 
TABLE 3.3. Approximations (3.1) to the sum of (1.2) for $a=i \alpha$, $\alpha>0, b=0, \nu=\frac{1}{2}, m=1$

$\begin{array}{cccc}n & \alpha=1 & \alpha=8 & \alpha=64 \\ 5 & 2.0061526550 & 0.782147849843 & 0.27762942965433 \\ & -0.796488122 & -0.6029037623 & -0.254862241652 \\ 10 & 2.006152655226 & 0.78214784984205 & 0.27762942965430958 \\ & -0.7964881235693 & -0.6029037624090 & -0.25486224165720 \\ 15 & 2.00615265522740 & 0.782147849842075 & 0.27762942965430952 \\ & -0.796488123569842 & -0.60290376240912464 & -0.2548622416572145 \\ 20 & 2.006152655227413 & 0.782147849842075 & 0.27762942965430952 \\ & -0.796488123569847 & -0.60290376240912460 & -0.25486224165721467 \\ 25 & 2.00615265522741422 & 0.78214784984207493 & 0.27762942965430952 \\ & -0.79648812356984801 & -0.60290376240912467 & -0.25486224165721468 \\ 30 & 2.00615265522741426 & 0.78214784984207492 & \\ & -0.79648812356984802 & -0.60290376240912468 & \end{array}$

TABLE 3.4. Approximations (3.1) to the sum of (1.2) for $a=$ $1+b+\omega i, \omega>0, b=\frac{1}{2}, \nu=\frac{9}{10}, m=1$

$\begin{array}{cccc}n & \omega=1 & \omega=8 & \omega=64 \\ 5 & 9.27126436493 & 8.1408914758952 & 6.625908132988981 \\ & -0.3966333402 & -1.05441141121 & -1.0214791502713 \\ 10 & 9.2712643649515 & 8.14089147589500 & 6.62590813298898329 \\ & -0.3966333403072 & -1.05441141122257 & -1.021479150271591 \\ 15 & 9.271264364951661 & 8.140891475895024 & 6.62590813298898329 \\ & -0.396633340307402 & -1.05441141122257 & -1.02147915027159224 \\ 20 & 9.2712643649516653 & 8.140891475895024 & 6.62590813298898329 \\ & -0.3966333403074054 & -1.0544114112225691 & -1.02147915027159226 \\ 25 & 9.27126436495166560 & 8.14089147589502396 & \\ & -0.39663334030740557 & -1.05441141122256908 & \\ 30 & 9.27126436495166563 & 8.14089147589502394 & \\ & -0.39663334030740558 & -1.05441141122256909 & \end{array}$

no difficulties are observed in case of large $\alpha$. The same accuracy is obtained for other values of $a, b, \nu$, and $m$; Table 3.4 gives the results obtained by the proposed method for $a=1+b+\omega i, \omega>0, b=\frac{1}{2}, \nu=\frac{9}{10}$, and $m=1$.

\section{ACKNOWLEDGMENTS}

Professor Walter Gautschi has kindly communicated to the author that the use of the Euler-Maclaurin formula for the special case $b=0, a=m=1$, $\nu=\frac{1}{2}$ of the series (1.2) has been previously suggested by Professor George M. Phillips at the University of St. Andrews (see [2, pp. 40-41]).

The author wishes to express his gratitude to the referees for their helpful comments and suggestions which have improved the original manuscript.

\section{BIBLIOGRAPHY}

1. M. Abramowitz and I. A. Stegun (eds.), Handbook of mathematical functions, National Bureau of Standards, Washington, DC, 1964.

2. P. J. Davis, Spirals: from Theodorus to chaos, A K Peters, Wellesley, MA, 1993. 
3. W. Gautschi, A class of slowly convergent series and their summation by Gaussian quadrature, Math. Comp. 57 (1991), 309-325.

4. I. S. Gradshteyn and I. M. Ryzhik, Tables of integrals, series and products, Academic Press, New York, 1980.

Institute of Computer Science, University of Wroclaw, ul. Przesmyckiego 20, 51-151 Wroclaw, Poland

E-mail address: sle@ii.uni.wroc.pl 